\title{
Maintenance and Characterization of New Exotic Inbred Line of Maize
}

\author{
Monir Hosen ${ }^{1}$, Mst. Farhana Nazneen Chowdhury ${ }^{2}$, M. Shalim Uddin ${ }^{3}$, Ratan \\ Kumar Paul ${ }^{4}$, Debu Kumar Bhattacharjya ${ }^{2}$ \\ ${ }^{1}$ Instructor, Agricultural Training Institute, Sher-e-Bangla Nagar, Dhaka-1207, Bangladesh \\ ${ }^{2}$ Department of Biochemistry, Sher-e-Bangla Agricultural University, Dhaka-1207, Bangladesh \\ ${ }^{3}$ Senior Scientific Officer, Genetices \& Plant Breeding Division, BARI, Gazipur, Bangladesh \\ ${ }^{4}$ Department of Chemistry, Bangladesh University of Engineering \& Technology, Dhaka-1000, Bangladesh
}

\begin{abstract}
One hundred and forty six inbred lines CIMMYT maize were characterized based on some morphological traits and grain yield at the experimental field of Bangladesh Agricultural Research Institute (BARI) during 2009-10 to study the maintenance and characterization of new exotic maize inbreeds. Maximum variation was found in number of grains per row, number of cobs per row, grain yield/plant, cob length (cm), ear height (cm) and plant height (cm). On the basis of D2 analysis, 146 inbred lines were grouped into 6 clusters. The inter cluster D2 values varied from 0.974 to 6.595 which exhibited medium range of diversity present in the inbred lines. It is expected that crossing of inbred lines belonging high to medium D2 values tend to produce high heterosis for yield. Development of hybrids utilizing these genotypes has the chance to obtain higher heterosis with high performing crosses. In order to achieve more heterotic partners from 146 CIMMYT (India) inbreeds were characterized for their morphological traits and kernel yield. Selfed seeds for each of the inbreeds line also maintained for future breeding works. Development of hybrids utilizing these genotypes has the chance to obtain higher heterosis with high performing crosses.
\end{abstract}

Keywords: Characterization, Cluster Analysis, Inbred Lines, Zea mays L

\section{Introduction}

Maize (Zea mays L.) is currently produced on nearly 100 million hectares in 125 developing countries and is among the three most widely grown crops in 75 of those countries FAOSTAT (2010) [1]. Between now and 2050, the demand for maize in the developing world will double, and by 2025 , maize production is expected to be highest globally, especially in the developing countries [2]. Maize (Zea mays L.) has become an important cereal crop in the rice based cropping system of Bangladesh. Another important challenge that threatens the long-term production growth of maize is the changing global climate [3]. Climate change scenarios show agriculture production will largely be negatively affected and will impede the ability of many regions to achieve the necessary gains for future food security [4]. However, maize-based diets, particularly those of the very poor, often lack essential vitamins and minerals. Over 50 million people in these regions are vitamins A deficient, which can lead to visual impairments, blindness and increased child mortality. The good news is that there is tremendous genetic variation in maize resulting in variable concentrations of provitamin A.

The project "Biofortified Maize for Improved Human Nutrition" at CIMMYT has been screening maize samples, looking for the best provitamin A content. Scientists anticipate producing materials with the ultimate target of $15 \mu \mathrm{g} / \mathrm{g}$ within the next four years by using cutting edge lab tools to help select the best materials for breeding. Maize inbred lines represent a fundamental resoures for genetics breeding while maize inbreeds are used extensively in hybrid corn production [5]. Hybrid maize has higher yield potentiality than those of synthetics and composites [6,7]. The hybrid varieties which are currently planted throughout the country are imported from India, China and Thailand etc. The most limiting factor of maize research in Bangladesh is the development, improvement and maintenance of parental/inbred lines. On the other hand, the problem of imported hybrid seed is the involvement of high price and uncontrolled quality. Moreover, the farmers cannot get the seeds timely. One important approach to improve this situation is the development of inbred lines which can produce high yielding hybrid varieties. Before hybrid development, prospective parent (inbred line) selection is a pre-requisite.

The genetic diversity between the genotypes is important as the genetically diverged parents are able to produce high heterotic effects [8-11]. Maize breeders are consistently emphasizing the importance of diversity among parental genotypes as a significant factor contributing to heterotic hybrids [12]. Characterization of genetic diversity of maize germplasm is of great importance in hybrid maize breeding [13]. $\mathrm{D}^{2}$ analysis is a useful tool for quantifying the degree of divergence between biological population at genotypic 
level and in assessing relative contribution of different components to the total divergence both intra and intercluster level [14-16].

Presently, the maize acreage and production scenario of Bangladesh are very encouraging due to cultivation of high yielding hybrids by the farmers. Farmers are also getting more profit from hybrid maize compared to rice and wheat. Inbred lines are the prerequisite for hybrid seed production. For developing high yielding hybrids large number of inbred lines should be evaluated with their diverged gene pool. The purpose of this study is to highlight the enormous genetic diversity in maize inbreeds and need for novel and systemic initiatives to understand and utilize the genetic diversity being genetically diverse, adapted and associated with traditional farming system [17].

\subsection{Location of Study}

\section{Material and Methods}

Seeds of maize inbreeds line were received from CIMMYT, India. The study was conducted during December 2009 to May 2010 at the experimental field of the Genetics and Plant Breeding Division, Bangladesh Agriculture research Institute, Joydebpur, Gazipur, Bangladesh.

\subsection{Plant Materials}

This study was a part of a long-term maize inbred line development program conducted at the BARI mentioned above where near homozygous inbred lines were developed from single cross hybrid varieties as source of population.

\subsection{Experimental design, plot arrangement, cultivation practices and data collection}

146 maize inbred lines were grown in alpha lattice design with three replications during rabi season of 2009-2010. The seeds of each were sown on 8 December 2009 in $5 \mathrm{~m}$ length one row with spacing of $0.75 \mathrm{x}$ $0.20 \mathrm{~m}$ between rows and hills, respectively. One plant was kept per hill after proper thinning. Recommended doses of fertilizers were applied. The other intercultural operations were done timely and properly to raise the crop uniformly. Observations were recorded on whole plot basis for days to 50\% tasseling (DT) and 50\% silking (DS). Ten randomly selected plants were used for recording observations on plant height (PH), ear height (EH), cob length (CL), cob diameter (CD), number of cobs per row (CPR), number of grains/cobs (GPC), 1000-grain weight (g) (TGW) and grain yield/plant (g) (GPP). Data were recorded according to maize descriptor during vegetative, flowering, harvesting and post harvesting periods. At flowering stage healthy disease free plants were selected and also selfed by hand for maintenance of desirable lines. The mean of the 10 plants were subjected to statistical analysis. Genetic diversity was estimated as per Mahalanobis $D^{2}$ Statistics (1936) and Clustering of genotypes was done according to Tochers method as described by Rao(1952) [18].

\section{Results And Discussion}

3.1 Characteristics of exotic inbred lines of maize

Range, mean, standard deviation and co-efficient of variation of different 10 characters of the studied inbred lines is presented in Table 1. Maximum variation was found in plant height $(\mathrm{cm})$, grain yield/plant $(\mathrm{gm})$, cob diameter $(\mathrm{cm})$, number of grains per row, and cob length $(\mathrm{cm})$. Hence there is enough scope for selection of potential inbred lines for hybridization program. Similar results have also been reported by P. C. Mahalanobis et al. (1981) [19], D. S. Falconer et al. (1960) [20] and V. Arunachalam et al. (1936) [21].

Table 1. Characteristics of exotic inbred lines of maize at BARI, Joydebpur during rabi (2009-10)

\begin{tabular}{|c|c|c|c|c|c|}
\hline \multirow[t]{2}{*}{ Characteristics } & \multirow[t]{2}{*}{ Mean } & \multicolumn{2}{|c|}{ Range } & \multirow[t]{2}{*}{ Standard Deviation } & \multirow[t]{2}{*}{$\mathrm{CV} \%$} \\
\hline & & Min & Max & & \\
\hline 1. Days to $50 \%$ tasseling & 84.98 & 68.0 & 105.0 & 7.42 & 8.73 \\
\hline 2. Days to $50 \%$ silking & 98.18 & 72.0 & 108.0 & 7.82 & 8.77 \\
\hline 3. Plant height $(\mathrm{cm})$ & 30.06 & 6.5 & 84.6 & 14.26 & 47.43 \\
\hline 4. Ear height $(\mathrm{cm})$ & 92.89 & 41.5 & 158.0 & 21.08 & 22.70 \\
\hline 5. Cob length $(\mathrm{cm})$ & 11.01 & 5.0 & 22.0 & 3.00 & 27.30 \\
\hline 6. Cob diameter $(\mathrm{cm})$ & 11.86 & 5.5 & 22.0 & 3.46 & 29.16 \\
\hline 7. Number of cob per rows & 10.95 & 4.0 & 15.30 & 1.86 & 17.02 \\
\hline 8. Number of grains percob & 15.21 & 3.0 & 27.0 & 4.39 & 28.84 \\
\hline 9. 1000-seed weight & 23.71 & 12.0 & 31.0 & 4.24 & 17.89 \\
\hline 10. Grain yield / plant (g) & 40.92 & 2.16 & 105.84 & 18.70 & 45.71 \\
\hline
\end{tabular}




\subsection{Cluster Analysis}

By application of non-hierarchical clustering using co-variance matrix, 146 inbred lines of maize were grouped into six different clusters (Table 2). It was revealed that cluster VI comprised maximum number (30) of genotypes, followed by cluster I and cluster III comprising 28 and 25 genotypes, respectively.

Table 2. Distribution of 146 maize inbred lines in six different clusters

\begin{tabular}{|c|c|c|}
\hline Cluster & No. of inbreds & Inbred lines included in different clusters \\
\hline I & 28 & $\begin{array}{l}\text { P31C4S5B-23-\#-\#-4-BBB-B-B-B-B-4-B, P31C4S5B-6-\#-\#-B-B-B-B-B-B-B-B-8-B, P31C4S5B-99-JMM- } \\
\text { B-B-B-B-B-B-B-B-3-B, CA03141-1-B-2-B, CA14514-5-B-2-B, CA14514-7-B-2-B, CA14514-8-3-2-B, } \\
\text { CA14514-9-4-2-B, CA14514-9-6-3-B, CA14514-B-2-B-2-B, CA00102-B-1-B-2-B, CA03118-1-B-2-B, } \\
\text { CA03118-B-4-4-2-B, CA03118-B-6-, 2-1-B, CA03130-1-2-B-3-B, CA03130-B-B-3-B-1-B, P31C4S5B- } \\
\text { 85-\#\#-1-4-5-BBB-B-B-B-1-B-2-B, Pop.31DMR-88-3\#-B*13-B-B-2-B-2-B, CML72-1-B, CML113-1-B, } \\
\text { CML161-2-B, CML290-2-B, CML326-1-B, CML421-2-B, CML425-1-B, CML426-2-B, CML439-2-B }\end{array}$ \\
\hline II & 22 & $\begin{array}{l}\text { CA14701-B-B-1-B, P147-F2\#105-2-1-B-1-B-B-B-B-B-B-B-2-B, Pop.31C4S5B-85-\#-\#-1-2-B-B-B-B-B- } \\
\text { B2-B-B-4-B, SW92145-2EV-7-3-B-B-B-B-B-B-B-B-4-B, P31C4S5B-38-\#-\#-3-B-B-B-B-B-B-2-B, } \\
\text { CA14514-2-1-2-B, CA14514-2-6-2-B, CA14514-4-1-1-B, CA14514-4-3-1-B, CA03120-1-B-1-B, } \\
\text { P31C4S5B-33-\#-\#-11-BBBB-B-B-B-2-B-3-B, CML162-1-B, CML165-1-B, CML167-2-B, CML170-1-B, } \\
\text { CML171-2-B, CML187-1-B, CML287-1-B, CML328-2-B, CML422-2-B, CML423-2-B P31C4S5B-33-\#- } \\
\text { \#-8-B-B-B-B-B-B-B-3-B }\end{array}$ \\
\hline III & 25 & $\begin{array}{l}\text { CA003134-B-B-B-2-B, Pop.31C4S5B-6-\#-\#-1-2-B-B-B-B-B-B1-B-B-2-B, CA14502-B-B-B-2-B, } \\
\text { CA03139-6-4-2-B, CA14514-3-B-1-B, CA14709-B-2-B-1-B, CA03130-1-5-B-1-B, CA03130-B-B-2-B-1- } \\
\text { B, CML425-2-1-3-B, P31C4S5B-33-\#-\#-11-BBBB-B-B-3-B-1-B, P31C4S5B-39-\#-\#-B-B-B-B-1-B-1-B, } \\
\text { P31C4S5B-39-\#-\#-B-B-B-B-3-B-1-B, P31C4S5B-39-\#-\#-B-B-B-B-4-B-2-B, P31C4S5B-39-\#-\#-B-B-B-B- } \\
\text { 6-B-2-B, P31C4S5B-39-\#-\#-B-B-B-B-B-8-6-3-B, P31C4S5B-23-\#-\#-6-BBBB-B-B-1-4-2-B, P31C4S5B- } \\
\text { 23-\#-\#-6-BBBB-B-B-1-7-2-B, CML116-1-B, CML172-2-B, CML284-2-B, CML291-1-B, CML336-1-B, } \\
\text { CML338-1-B, CML468-2-B, P31C4S5B-33-\#-\#-11-B-B-B-B-B-B-B-B-3-B, CA03141-2-B-1-B }\end{array}$ \\
\hline IV & 18 & $\begin{array}{l}\text { CA03141-3-B-2-B, SW92145-2EV-7-3-B-B-B-B-B-5-B-1-B, P31C4S5B-39-\#-\#-B-B-B-B-B-8-8-2-B, } \\
\text { P31C4S5B-23-\#-\#-6-BBBB-B-B-3-B-2-B, P31C4S5B-41- \#-\#-B-B-B-B-B-B-1-B-1-B, P31C4S5B-6-\#-\#- } \\
\text { 1-1-B-B-B-B-B-4-B-2-B, P31C4S5B-6-\#-\#-1-1-B-B-B-B-B-5-4-2-B, CML472-B-B-B-3-B, CML30-2-B, } \\
\text { CML112-1-B, CML122-1-B, CML292-3-B, CML412-1-B, CML436-1-B, CML465-2-B, CML479-2-B, } \\
\text { CML480-1-B, CA03139-B-B-3-B, CA14514-1-B-1-B, CA03139-B-B-3-B, CA14514-1-B-1-B }\end{array}$ \\
\hline $\mathrm{V}$ & 23 & $\begin{array}{l}\text { CA14514-B-1-B-2-B, CA14514-B-3-B-2-B, P31C4S5B-39-\#-\#-B-B-B-B-B-7-B-3-B, P31C4S5B-39-\#-\#- } \\
\text { B-B-B-B-B-8-3-2-B, P31C4S5B-39-\#-\#-B-B-B-B-B-8-7-2-B, CA14709-4-7-5-1-B, P31C4S5B-6-\#-\#-1-1- } \\
\text { B-B-B-B-B-6-B-1-B, CML469-1-B-B-2-B, CML33-1-B, CML41-2-B, CML51-2-B, CML58-1-B, CML70- } \\
\text { 2-B, CML71-2-B, CML298-2-B, CML307-1-B, CML337-1-B, CML413-2-B, CML433-2-B, CML454-2- } \\
\text { B, CML481-1-B CA00106-B-B-B-5-B, P31C4S5B-39-\#-\#-B-B-B-B-B-4-B-2-B, P31C4S5B-39-\#-\#-B-B- } \\
\text { B-B-B-8-5-2-B }\end{array}$ \\
\hline VI & 30 & $\begin{array}{l}\text { P31C4S5B-39-\#-\#-B-B-B-B-B-8-9-2-B, P31C4S5B-23-\#-\#-6-BBBB-B-B-5-B-2-B, P31C4S5B-41- \#-\#-B- } \\
\text { B-B-B-B-B-2-B-2-B, P31C4S5B-6-\#-\#-1-1-B-B-B-B-B-5-1-2-B, P31C4S5B-6-\#-\#-1-1-B-B-B-B-B-5-2-2- } \\
\text { B, P31C4S5B-23-\#-\#-4-BBB-B-B-B-B-B-4-B, CML 421-B-B-B-3-B, CML 425-B-B-B-4-B, CML 470-B- } \\
\text { B-B-3-B, CML-474-B-B-B-2-B, CML20-2-B, CML28-2-B, CML162-1-B, CML193-2-B, CML412-1-B } \\
\text { CML412-1-B, CML293-1-B, CML294-1-B, CML296-1-B, CML299-1-B, CML300-1-B, CML287-1-B, } \\
\text { CML304-1-B, CML359-2-B, CML360-1-B, CML424-1-B,CML434-2-B, CML435-2-B, CML466-2-B, } \\
\text { CML487-2-B, CML496-1-B }\end{array}$ \\
\hline
\end{tabular}

The lowest genotype was included in cluster IV (18). Clustering pattern of inbred lines under this study reveals that the inbred lines showed considerable genetic diversity among themselves by occupying six different clusters. Similar results were reported by Singh et al. (2005) [22] and Liu YuAi et al. (2006) [23] in maize and by Masud et al. (1995) [24] in pumpkin. Another study was carried out by Chen FaBo et al. (2007) [25] who reported that 186 maize genotypes could be classified into ten clusters. On the basis of $\mathrm{D}^{2}$ analysis, 146 inbred lines were grouped into six clusters (Table 2). Clustering pattern of inbred lines under this study reveals that the inbred lines showed considerable genetic diversity among themselves by occupying six different clusters. Present investigation was supported by Singh et al. (2005) [22] and Liu YuAi et al. (2006) [23] in maize and by Masud et al. (1995) [24] in pumpkin. Another study was carried out by Chen FaBo et al. (2007) [25] who reported that 186 maize genotypes could be classified into ten clusters.

The maximum inter cluster distance was observed between cluster II and IV (Table 3) indicating that the inbred lines grouped in these clusters were highly divergent from each other. The intra cluster $\mathrm{D}^{2}$ value was the least in cluster I and the highest in cluster IV. $\mathrm{D}^{2}$ values varied from 0.974 to 6.595 exhibited high to medium range of diversity presence in the inbred line and principal component scores also indicated a high 
degree of genetic diversity among the genotypes.

The maximum inter-cluster divergence (Table 3) was observed between the clusters II and IV and it was minimum between clusters I and V. The maximum intra-cluster distance was observed in cluster IV and minimum in cluster I. The crosses involving parents from most divergent clusters are expected to manifest maximum heterosis and generate wide variability in genetic architecture. Intra cluster distance was much lower than the inter cluster one, suggesting, heterogeneous and homogeneous nature between and within groups, respectively.

Table 3. Inter and intra-cluster (bold) distance $\left(\mathrm{D}^{2}\right)$ for 146 maize inbred lines obtained by canonical variate analysis

\begin{tabular}{lllllll}
\hline Cluster & I & II & III & IV & V & VI \\
\hline I & $\mathbf{0 . 1 2 3}$ & & & & & \\
II & 2.212 & $\mathbf{0 . 5 4 2}$ & & & & \\
III & 3.307 & 4.346 & $\mathbf{0 . 4 8 7}$ & & & \\
IV & 4.413 & 6.595 & 4.321 & $\mathbf{1 . 3 4 5}$ & & \\
V & 0.974 & 2.535 & 4.235 & 4.582 & $\mathbf{0 . 7 5 6}$ & \\
VI & 4.009 & 6.029 & 5.925 & 3.008 & 3.500 & $\mathbf{1 . 2 3 8}$ \\
\hline
\end{tabular}

This was further supported by an appreciable variation observed for cluster means (Table 4). Mean values of Plant height (PH), Ear height (EH), Cob length (CL), number of cob per row (CPR), number of grains per cob (GPC) and Grain yield /plant (GYP) were the highest in cluster IV; Cob diameter (CD) and 1000-seed weight in cluster III. A wide range of variations for several characters among single as well as multigenotypic cluster was observed. However, the difference was clear for plant height, ear height, cob length, number of cob per row, number of grains per cob and grain yield /plant, which has contributed largely to the total divergence.

Table 4. Cluster means for 10 different characters of 146 maize inbred lines

\begin{tabular}{|c|c|c|c|c|c|c|}
\hline \multirow[t]{2}{*}{ Characters } & \multicolumn{6}{|c|}{ Clusters } \\
\hline & I & II & III & IV & $\mathrm{V}$ & VI \\
\hline 1. Days to $50 \%$ tasseling & 79.37 & 80.29 & 79.80 & 86.68 & 91.91 & 91.23 \\
\hline 2. Days to $50 \%$ silking & 83.37 & 85.48 & 83.16 & 90.58 & 96.52 & 95.50 \\
\hline 3. Plant height (cm) & 23.17 & 16.99 & 21.99 & 45.39 & 27.84 & 44.10 \\
\hline 4. Ear height $(\mathrm{cm})$ & 89.51 & 65.68 & 82.94 & 118.46 & 80.96 & 116.24 \\
\hline 5. Cob length $(\mathrm{cm})$ & 10.25 & 9.55 & 12.19 & 12.32 & 10.76 & 11.10 \\
\hline 6. Cob diameter $(\mathrm{cm})$ & 11.33 & 10.70 & 13.94 & 12.49 & 10.73 & 11.88 \\
\hline 7. Number of cob per row & 9.69 & 10.28 & 12.22 & 12.48 & 10.73 & 10.68 \\
\hline 8. Number of grains per cob & 12.03 & 10.96 & 18.75 & 19.58 & 14.87 & 15.57 \\
\hline 9. 1000-seed weight & 23.26 & 22.90 & 28.32 & 25.32 & 22.30 & 20.90 \\
\hline 10. Grain yield /plant (g) & 27.84 & 25.59 & 65.07 & 61.32 & 35.72 & 34.35 \\
\hline
\end{tabular}

Similar results have also been reported by Singh et al. (2005) [22] and Chen FaBo et al. (2007) [25]. Hence, for the improvement of different characteristics viz; Plant height, Ear height, Cob length, Number of cob per row, Number of grains per cob and Grain yield/plant under the present study, inbred lines should be selected form cluster IV and III.

Table 5. Relative contributions of the 10 characters to the total divergence in maize

\begin{tabular}{lcc}
\hline Characters & Vector I & Vector II \\
\hline 1. Days to 50\% tasseling & 0.03167 & 0.03377 \\
2. Days to 50\% silking & 0.03162 & 0.04827 \\
3. Plant height $(\mathrm{cm})$ & 0.02547 & 0.01466 \\
4. Ear height $(\mathrm{cm})$ & 0.08318 & -0.01677 \\
5. Cob length $(\mathrm{cm})$ & 0.02094 & -0.02406 \\
6. Cob diameter $(\mathrm{cm})$ & 0.05021 & 0.01137 \\
7. Number of cob per row & 0.13902 & 0.30954 \\
8. Number of grains per cob & 0.14579 & 0.20078 \\
9. 1000 seed weight & 0.00796 & 0.05951 \\
10. Grain yield / plant $(\mathrm{g})$ & -0.03817 & -0.15556 \\
\hline
\end{tabular}


In Table 5, the principal component analysis revealed that in major vector I, the important characters responsible for genetic divergence in the major axis of differentiation were days to $50 \%$ tasseling, days to $50 \%$ silking, plant height, cob diameter, cob length, number of rows per cob, number of grains per row, 1000-seed wt. In vector II, which was the second axis of differentiation, the characters, days to $50 \%$ tasseling, days to $50 \%$ silking, plant height, cob diameter, number of rows per cob, number of grains per row, 1000-seed wt important. The role of these parameters for both of the vectors was positive across two axis which is the indication of the important components of genetic divergence among the inbreeds.

\section{Conclusion}

Maintenance and characterization of new exotic inbreed line was studied to find out the more diverse inbred lines in maize which might be used in hybridization programme. 146 maize inbred lines were grouped into six different clusters. The crosses involving parents/inbred lines from most divergent clusters are expected to manifest maximum heterosis and generate wide variability in genetic architecture.

\section{Acknowledgements}

I would like to gratefully acknowledged CIMMAT (India) for supply seed to study this research. The Genetics and Plant Breeding Division, Bangladesh Agriculture research Institute, Joydebpur, Gazipur is gratefully acknowledged for their kind cooperation regarding the provide field facilities and financial cooperation for cultivation of maize inbreeds.

\section{References}

[1] FAOSTAT, Statistical database and data-sets of the Food and Agriculture Organization of the united nation, 2010. (http://faostat.fao.org/default.aspx)

[2] M. R. Rosegrant, C. Ringler, T. B. Sulser, M. Ewing, A. Palazzo, T. Zhu, Etal. Agriculture and food security under global change, 2009: Prospects for 2025/2050 (Washington, D.C.: International Food policy Research Institute).

[3] J. E. Cairns, K. Sonder, P. H. Zaidi, N. Verhulst, G. Mahuku, R. Babu, S. K. Nair, B. Das, Maize production in a changing climate: impact, adaptation and mitigation strategies. Adv. Agron. 114, 2012, 1-58.

[4] D. B. Lobaell, M. B. Burk, C. Tebaldi, M. D. Mastrandre, W. P. Falcon, R. L. Nylor, Prioritizing climate change adaptation need for food securityin 2030. sci. 319, 2008, 607-610.

[5] Anderson, Edgar, L. B. William, The history of the common maize variety of the United States Corn Belt. Agricultural History 26, 1952, 2-8.

[6] S. Pandey, C. O. Gardner, Recurrent selection for population variety and hybrid improvement in tropical maize. Advances in Agronomy, 48, 1992, 1-87.

[7] S. K. Vasal, B. S. Dhillon, G. Srinivasan, S. D. McLean, J. Crossa, S. H. Zhang, Improvement in selfed and random-mated generations of four subtropical maize populations through $\mathrm{S}_{3}$ recurrent selection. Euphytica, 1995 - Springer, 83, 1995, 1-8.

[8] D. S. Falconer, Introduction to quantitative genetics. Oliver \& Boid, London, 1960, 304p.

[9] V. Arunachalam, Genetic distance in plant breeding. Indian Journal of Genetics, 41, 1981, 226-236.

[10] A. Ghaderi, M. Shishegar, A. Regai, B. Ehdaie, Multivariate analysis of genetic diversity for yield and its components in mungbean. Journal of American Society of Horticultural Science, 104, 1984, 728-731.

[11] M. A. K. Mian, P. N. Bahl, Genetic divergence and hybrid performance in chickpea. Indian Journal of Genetics, 49, 1989, 119-124.

[12] B. S. Ahloowalia, N. I. Dhawan, Effect of genetic diversity in combining ability of inbred lines of maize. Indian Journal of Genetics, 23, 1963, 158-162.

[13] X. C. Xia, J. C. Reif, A. E. Melchinger, M. Frisch, D. A. Hoisington, D. Beck, K. Pixley, M. L. Warburton, Genetic diversity among CIMMYT maize inbred lines investigated with SSR markers. Crop Science, 45, 2005, 2573-2582.

[14] B. R. Murty, V. Arunachalam, The nature of divergence in relation to breeding system in crop plants. Indian Journal of Genetics, 26A, 1966. 188-198.

[15] J. Ram, D. V. S. Panwar, Interspecific divergence in rice (Oryza sativa L.). Indian Journal of Genetics, 30, $1970,1-2$.

[16] K. S. Sachan, J. R. Sharma, Multivariate analysis of divergence in tomato. Indian Journal of Genetics, 31, $1971,86-93$.

[17] T. C. C.-Villa, N. Maxted, M. Scholten, B. F.-Lloyd, Defining and identifying crop landraces. Plant Genet. Res. 33, 2005, 73-384.

[18] C. R. Rao, Advanced Statistical Methods in Biometrical Research. John Wiley and Sons, New York, 1952,390 pp.

[19] V. Arunachalam, Genetic distance in plant breeding. Indian Journal of Genetics, 41, 1981, 226-236.

[20] D. S. Falconer, Introduction to quantitative genetics. Oliver \& Boid, London, 1960, 304p.

[21] P. C. Mahalanobis, On the generalized distance in statistics. Proceedings National Institute of Science, India, 2(1), 1936, 49-55.

[22] P. Singh, D. Sain, V. K. Dwivedi, Y. Kumar, O. Sangwan, Genetic divergence studies in maize (Zea mays L.). Annals of Agricultural and Biological Research, 10(1), 2005, 43-46.

[23] L. Y. Ai, H. J. Hua, G. Z. Jun, Z. Wei, Principal component analysis and cluster analysis of introduced maize varieties. Journal of Maize Science, 14(2), 2006, 16-18.

[24] M. A. T. Masud, M. A. Chowdhury, M. A. Hossain, S. M. M. Hossain, Multivariate analysis in pumpkin (Cucurbita moschata Duch ex. Poir). Bangladesh Journal of Plant Breeding and Genetics, 8(1\&2), 1995, 45-50.

[25] C. F. Bo, Y. K. Cheng, R. T. Zhao. P. G. Tang, Analysis of genetic diversity of maize hybrids in the regional tests of Sichuan and Southwest China. Acta Agronomica Sinica, 33(6), 2007, 991-998. 